The Astrophysical Journal, 274:L7-L11, 1983 November 1

๑ 1983. The American Astronomical Society. All rights reserved. Printed in U.S.A.

\title{
ALIGNMENT OF GALAXIES IN THE COMA CLUSTER
}

\author{
S. DJORGovsKI \\ Astronomy Department, University of California, Berkeley \\ Received 1983 May 5; accepted 1983 June 9
}

\begin{abstract}
Analysis of photometry data of the Coma Cluster galaxies shows a prominent effect of galaxy alignment with the cluster itself. The effect shows strong dependence on galaxy position within the cluster, galaxy luminosity, and, to a lesser degree, color. Galaxies also show a tendency to point toward their nearest neighbors. Quantitative analysis of these effects is presented. Independent measurements of position angles in a small subset of data have been performed. Possible spurious causes for anisotropy are discussed, and it is argued that the effect is real. Weak tendencies found by the earlier authors are in agreement with the results reported here. This effect gives strong support to the adiabatic (fragmentation) picture of galaxy and cluster formation.
\end{abstract}

Subject headings: cosmology — galaxies: clustering — galaxies: formation - galaxies: general

\section{INTRODUCTION}

Alignment of galaxies within clusters and superclusters is one of the crucial tests for theories of the largescale structure and galaxy formation. In this Letter, I present evidence for alignment of galaxies in the Coma Cluster with the cluster itself, as well as with the general orientation of the Coma/A1367 Supercluster.

The effects of coherent galaxy orientations have been sought by numerous authors. Brown $(1964,1968)$ claimed some evidence for preferred position angles (hereafter P.A.) of galaxies. His work was criticized by Hawley and Peebles (1975), who give the earlier references. Adams, Strom, and Strom (1980) have found a marginal tendency for alignment ( 0.068 probability of rejection level) in the A999 and A2197 Clusters, with the clusters' major axes. Thompson (1976) found the same effect in A2197 and showed that the effect is more prominent for the red galaxies. Strom and Strom (1978) discovered a tendency for alignment in the Perseus (A426) Cluster (rejection probability less than 0.05 ). They also found that the Perseus elliptical galaxies tend to be more flattened than field galaxies and those in spheroidal clusters. MacGillivray et al. (1982) found a tendency (probability level of 0.05 ) of $S$ and Irr galaxies in the Local Supercluster (LSC) to align with the LSC plane itself. Similar results were reported by Kaprandis and Sullivan (1983). Binggeli (1982) and Carter and Metcalfe (1980) discovered that some central cluster galaxies (typically cD's) tend to align with their clusters, though this is likely to be an evolutionary rather than a primordial effect.

The dynamics of the Coma Cluster was investigated by Rood et al. (1972) and Kent and Gunn (1982), who give the earlier references. Investigation of the ellipticity of Coma has been done by Schipper and King (1978). The cluster is elongated roughly in the E-W direction and has an ellipticity $\varepsilon \approx 0.5$. Gregory and Thompson (1978) demonstrated the connection of Coma with the A1367 Cluster, off to the west.

\section{DATA}

The data come from Godwin, Metcalfe, and Peach (1983). They consist of two-color ( $b$ and $r$ ) photometry of 6724 galaxies, down to $b=21$, covering $6.92 \mathrm{deg}^{2}$, centered on the Coma cluster. Ellipticity and P.A. measurements are also provided; 4217 galaxies have $\varepsilon \geq 0.1$, and they are the sample used in the present investigation. The details of photometry and comparisons with earlier work are discussed by Godwin $e t$ al. It should be pointed out that these machine measurements of $\varepsilon$ and P.A. are objective; thus, no observer bias can be present, though an algorithm bias is possible.

There is indeed such a bias: measured P.A. values tend to accumulate near the angles which are multiples of $45^{\circ}$. This anisotropy is probably due to the small numbers of pixels enclosed for the faint galaxies (which are in the majority). Important as it is, this effect is not detrimental to further analysis. It can be easily separated out, as shown in the next section.

Background estimates come from the models of Rainey and Abell (Abell 1977) and galaxy counts by Koo (1981), with appropriate color transformations. The estimated cumulative background amounts to 398 galaxies per square degree down to $b=21$, or 190 galaxies down to $b=20$. Assuming no differences in ellipticity between the cluster and the field galaxies, we can scale these numbers for the $\varepsilon \geq 0.1$ sample down to 250 and 119 respectively. 

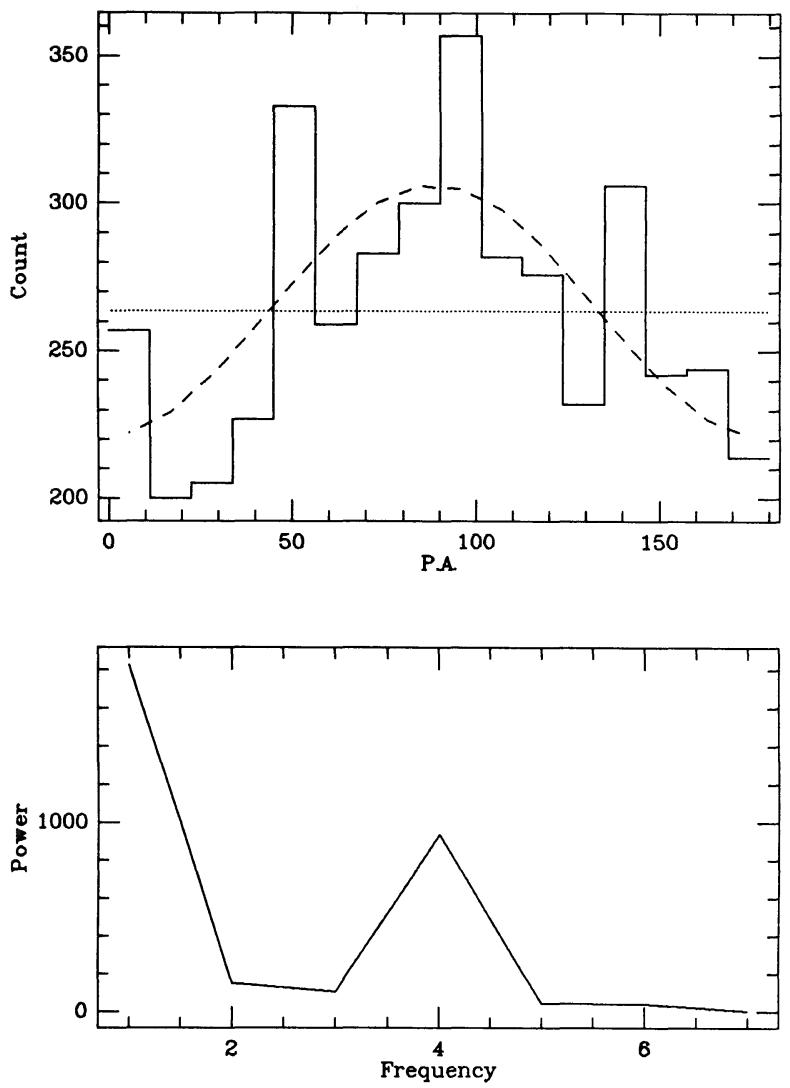

Fig. 1.-( upper) The histogram of position angles for all galaxies with $\varepsilon \geq 0.1$. The dotted line marks the mean expected value per bin, and the dashed line gives the best fit wave (from the components of the Fourier spectrum). (lower) The power spectrum of the histogram above.

\section{ALIGNMENT EFFECT}

In order to search for any preferred orientation of the Coma galaxies, 16 bin P.A. histograms were accumulated. The result for the whole $\varepsilon \geq 0.1$ sample is shown in Figure 1. Preference for P.A. values around $90^{\circ}$ is obvious. A picket-like periodicity of $45^{\circ}$ is also apparent, superposed on the global wave pattern. The bottom graph shows the Fourier power spectrum for this histogram. High power at the first frequency conveys the global wavelike anisotropy, whereas the peak at the fourth frequency reflects the $45^{\circ}$ periodicity.

There are two ways of addressing this problem quantitatively, as discussed in detail by Hawley and Peebles (1975). First, one can accumulate the $\chi^{2}$ value corresponding to the null-hypothesis (isotropy):

$$
\chi_{\text {iso }}^{2}=\sum_{i}^{16} \frac{\left(N_{i}-N_{0}\right)^{2}}{N_{0}},
$$

where $N_{i}$ is the count in $i$ th bin, and $N_{0}$ the expected (mean) value. The probability $P_{\text {iso }}$ corresponding to this $\chi^{2}$ value gives the level at which the isotropy hypothesis can be rejected. The second method is based on the wave model for the data:

$$
\begin{aligned}
N_{i}=N_{0} & {\left[1+\Delta_{11} \cos \left(2 \theta_{i}\right)\right.} \\
& \left.+\Delta_{12} \sin \left(2 \theta_{i}\right)+\cdots+\Delta_{82} \sin \left(16 \theta_{i}\right)\right],
\end{aligned}
$$

where $\theta_{i}$ is the central bin angle. The single-wave periodicity has the relative amplitude:

$$
\Delta_{1}=\left(\Delta_{11}^{2}+\Delta_{12}^{2}\right)^{1 / 2} .
$$

Let $p(s)$ be the Fourier power spectrum of the histogram values $N_{i}$. Then,

$$
p(1)=N_{0}^{2} \Delta_{1}^{2} .
$$

Thus, by doing a Fourier transform, we can easily obtain the amplitude of anisotropy, $\Delta_{1}$. The variance of this quantity, scaled appropriately, is

$$
\sigma^{2}\left(N_{0} \Delta\right)=16 N_{0}
$$

(the total number of galaxies). Thus we can estimate the probability that this wave comes from a random (isotropic) distribution:

$$
P\left(\Delta_{1}\right)=\exp \left[-0.5 \Delta_{1}^{2} / \sigma^{2}\left(\Delta_{1}\right)\right]=\exp \left[-8 p(1) / N_{0}\right] .
$$

Similarly, one can estimate the equivalent quantity for the $45^{\circ}$ periodicity, $P\left(\Delta_{4}\right)$. The power of this test lies in the fact that it separates these effects of different periodicity. Finally, one can obtain the P.A. of preferred orientation from

$$
\text { P.A. }(\max )=0.5 \operatorname{atan}\left(\Delta_{12} / \Delta_{11}\right) .
$$

Table 1 lists these quantities for the basic sample, and its various subdivisions. Also listed in the backgroundcorrected wave amplitude,

$$
\Delta_{1} \text { (b.c.) }=p(1)^{1 / 2} /\left[N_{0}(1-B)\right],
$$

where $B$ is the estimated (isotropic) background fraction.

The sample has been subdivided according to some other galaxian properties: the magnitude ("bright" and "faint"), $b=20$ being the dividing line; distance to the cluster center ("core," with $R_{\mathrm{cl}}<40$ ', and "envelope," with $40^{\prime}<R_{\mathrm{cl}}<70^{\prime}$ ); color ("blue" and "red"), ( $b-$ $r)=1.8$ being the dividing line; and position ("E-W" and "N-S"). In this last division, the whole area was divided into four triangular slices, and corresponding 
TABLE 1

STATISTICS FOR THE SAMPLES ${ }^{\mathrm{a}}$

\begin{tabular}{|c|c|c|c|c|c|c|c|c|}
\hline $\begin{array}{l}\text { Sample } \\
\text { (1) }\end{array}$ & $\begin{array}{l}N_{\text {gal }} \\
\text { (2) }\end{array}$ & $\begin{array}{l}\text { Est. Background } \\
\text { (\%) } \\
\text { (3) }\end{array}$ & $\begin{array}{l}\chi_{\text {iso }}^{2} \\
(4)\end{array}$ & $\begin{array}{c}\log P_{\text {iso }} \\
\text { (5) }\end{array}$ & $\begin{array}{c}\log P\left(\Delta_{1}\right) \\
(6)\end{array}$ & $\begin{array}{c}\Delta_{1} \text { (b.c.) } \\
\text { (7) }\end{array}$ & $\begin{array}{l}\text { P.A.(max) } \\
\text { (8) }\end{array}$ & $\begin{array}{c}\log P\left(\Delta_{4}\right) \\
\text { (9) }\end{array}$ \\
\hline$\varepsilon \geq 0.1$. & 4217 & $41 \%$ & 116.57 & -17.06 & -24.07 & 0.275 & 88.6 & -12.38 \\
\hline$\varepsilon \geq 0.2 \ldots \ldots \ldots$ & 3448 & $41 \%$ & 108.37 & -15.48 & -27.73 & 0.326 & 86.8 & -9.59 \\
\hline Core.......... & 1328 & $26 \%$ & 38.92 & -3.18 & -1.94 & 0.111 & 98.8 & -4.51 \\
\hline Envelope ....... & 1669 & $43 \%$ & 74.42 & -9.14 & -15.87 & 0.478 & 85.9 & -2.87 \\
\hline Bright $\ldots \ldots \ldots \ldots$ & 2283 & $36 \%$ & 75.07 & -9.29 & -9.42 & 0.215 & 92.7 & -10.09 \\
\hline Faint ... & 1934 & $47 \%$ & 61.70 & -6.89 & -15.79 & 0.366 & 85.1 & -5.50 \\
\hline Blue ..... & 1818 & $41 \%$ & 39.64 & -3.29 & -5.81 & 0.206 & 87.0 & -4.72 \\
\hline Red ..... & 2455 & $41 \%$ & 84.93 & -11.06 & -19.32 & 0.323 & 89.0 & -7.97 \\
\hline $\mathrm{N}-\mathrm{S} \ldots \ldots$ & 2095 & $41 \%$ & 88.52 & -11.73 & -16.52 & 0.323 & 87.4 & -8.63 \\
\hline E-W ........ & 2122 & $41 \%$ & 49.37 & -4.33 & -8.32 & 0.228 & 90.2 & -4.17 \\
\hline Neighbors & 4217 & $41 \%$ & 23.33 & -1.11 & -4.89 & 0.123 & $\ldots$ & $\ldots$ \\
\hline
\end{tabular}

${ }^{\mathrm{a}}$ See text for definitions of terms.

quadrants were lumped together. Figure 2 shows the histograms for two of these subsamples. Table 1 also lists the results for the "more elliptical" sample, with $\varepsilon \geq 0.2$, where P.A. measurements are likely to be more reliable.

One immediately notices from column (5) of Table 1 that none of the samples are isotropic. From column (6), which pertains to the degree of alignment, one notices several things: first, there is a strong dependence on the distance from the cluster center-the pointing directions of the core galaxies are less anisotropic; second, there is a prominent luminosity effect (the faint galaxies are more likely to be aligned); third, there is a color effect, in the sense that the red galaxies show the alignment more prominently (which is in agreement with the result of Thompson 1976); and fourth, there may be a stronger alignment tendency for the galaxies "above" and "below" the Coma plane (N-S sample). This last point is consistent with the findings of MacGillivray et al. (1982) for the LSC. Another thing to notice is that the $\varepsilon \geq 0.2$ sample shows the alignment effect more prominently than the $\varepsilon \geq 0.1$ sample; this may be due to better measurements of P.A. values for the more elongated images.

Lack of alignment in the core or for the bright galaxies, or both, can readily explain the negative result of Rood and Baum (1967), since they used the small number of brightest galaxies in the cluster core. The effect claimed by Thompson (1976), that the galaxies in Coma tend to point toward the cluster center, is probably a consequence of the alignment plus the elliptical shape of the cluster itself. I find that galaxies in the E-W sample indeed do tend to point toward the cluster center, but galaxies in the N-S sample tend to point perpendicularly to their radius vectors.

The next question is, Do the galaxies tend to point toward their nearest neighbors (in projection)? The
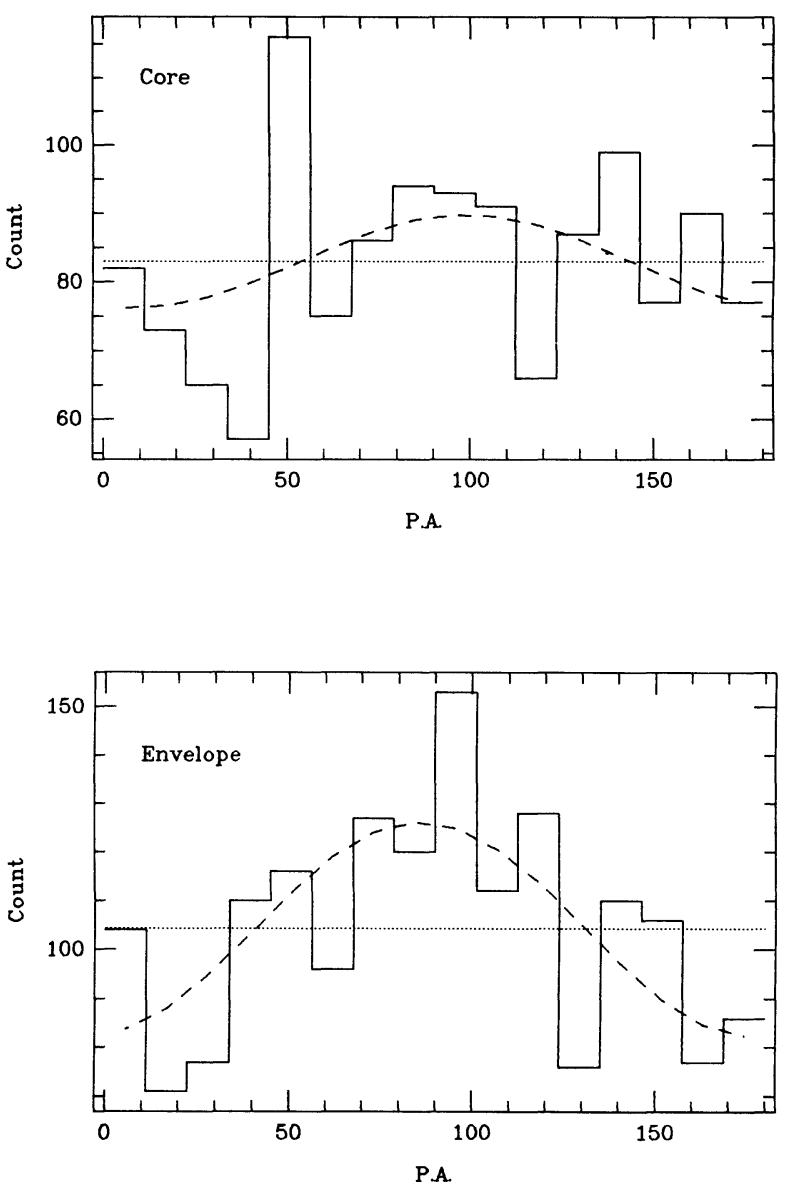

FIG. 2.-(upper) Same as Fig. 1, but for the cluster core selected sample. (lower) Same as Fig. 1, but for the cluster envelope selected sample. 


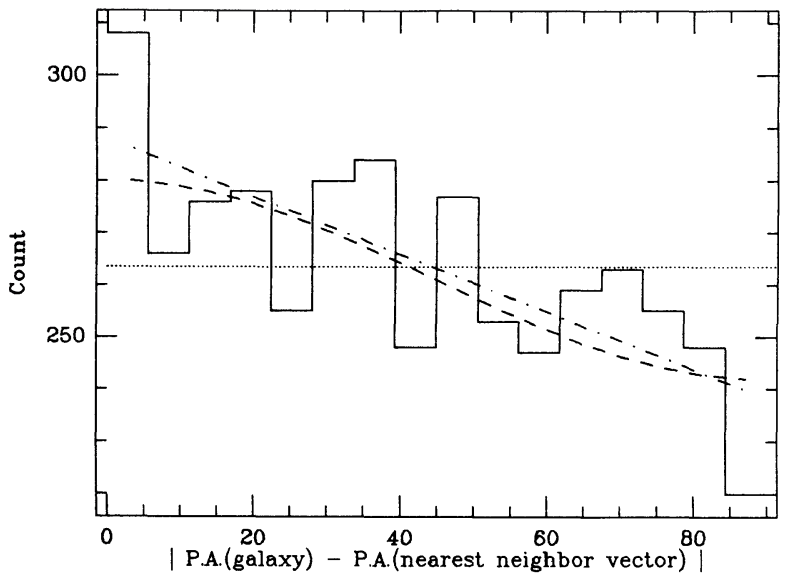

FIG. 3.-The histogram of position angle differences between a galaxy and the vector pointing toward its nearest neighbor. The dotted line is the expected mean; the dashed line is the best half-wave fit, as determined by the Fourier spectrum coefficients; and the dot-dashed line is the linear least squares fit.

answer is positive, and it is depicted in Figure 3, which shows the histogram of P.A. differences between the galaxy P.A. and the P.A. of the vector pointing toward its nearest neighbor. The statistic is listed in the last row of Table 1. The linear least squares fit to the bins is also an adequate representation.

I have also searched for the correlations of position angles of neighboring galaxies and for any alignment of galaxy-galaxy vectors. The results were negative, but one has to remember that projection can easily scramble any possible effects of this kind.

The alignment effects are clearly present in the data. Are they real? The Fourier test readily separated the $45^{\circ}$ anisotropy which is due, presumably, to the poor image sampling in intermediate directions. It is hard to imagine that any algorithm would show preference for the $X$-direction, and not for $Y$, and reproduce the radius, magnitude, color, position, and pointing effects. The same applies for possible scanning malfunctions. Uneven scan sampling in one direction would drastically reduce the number of $\varepsilon=0$ galaxies, which are about $38 \%$ of the total number. Telescope trailing errors also fail on all of those counts and should not perturb the outer isophotes anyway. Thus, the alignment effects in Coma appear to be real.

The ultimate test is, of course, repeatability of the measurement. I have obtained from $\mathrm{H}$. Spinrad a highscale $\left(99^{\prime \prime} 65 \mathrm{~mm}^{-1}\right)$ plate of the Coma Cluster core. The galaxies from the Godwin, Metcalfe, and Peach (1983) list have been identified and plate scanned with the Berkeley PDS microdensitometer. The scanning direction was at an angle of about $70^{\circ}$ with respect to the E-W direction, and the aperture was $00^{\prime \prime} 19$. The final sample consists of over 100 galaxies reaching down to $b \approx 19$. The results cannot be discussed at length here, but suffice it to say that there is good agreement between the two sets of measurements, and that there is no apparent systematic trend which could account for the $180^{\circ} \mathrm{E}-\mathrm{W}$ periodicity.

\section{IMPLICATIONS FOR GALAXY AND CLUSTER FORMATION}

The effects described here can be understood within the fragmentation (adiabatic) scenario of galaxy and cluster formation (see, e.g., Zeldovich 1978). The galaxies can inherit any dynamical anisotropies that may be present in the protocluster material-stellar motions in elliptical galaxies and bulges are fossils of the protogalactic dynamics. The adiabatic ("pancake") theory explicitly predicts that there will be little coherence in the pancake's (= present-day cluster's) central regions, which is what is observed. The luminosity dependence can be explained by the fact that the more luminous (larger) galaxies underwent more mergers of the protogalactic fragments, a process which may tend to scramble any anisotropy. The color effect may be understood as indicting that the old stellar populations remember those days better. The tendency for a galaxy to point toward its nearest neighbors is suggestive of coevality.

There is one possible disagreement. Doroshkevich and Shandarin (1974) and Shandarin (1974) predicted within this scenario that the galaxies will align perpendicularly to the cluster (pancake) plane. However, these authors did not explicitly solve this difficult problem, and their argument was primarily heuristic: colliding shock waves will create turbulence with vorticity vectors in the plane of the pancake. This may not be the most important effect. It is possible to imagine a scenario in which the fragments of a pancake acquire their angular momenta through mutual tidal torques. Oblate (weakly rotating) elliptical galaxies inherited their anisotropy directly from the protocluster material. And the possibility of filamentary collapse has not been investigated at all. The analysis of Doroshkevich and Shandarin was for a purely two-dimensional case, which Coma clearly is not. Another avenue which should be explored is the possibility that the primordial turbulence plays an important role in the process of galaxy formation (Ozernoi 1972, 1978; Doroshkevich 1973).

The hierarchical clustering (isothermal) scenario does not seem to provide any simple explanation for these coherence effects. Tidal distortions cannot account for them in a mere Hubble time (Palmer 1983) and would demand dependences on the galaxy location and luminosity that are the opposite of those observed. For most of the galaxies in the Coma envelope, the crossing time is quite comparable to the age of the universe.

Let us finally point out that some of the "background" galaxies may be legitimate members of the Coma/A1367 Supercluster or the foreground filament (NGC 4793 and NGC 4615 groups) and thus also show some alignment tendency. An interesting test would be 
to repeat this analysis for the galaxies in A1367 or the Perseus Cluster.

I am greatly indebted to Dr. J. Godwin for communicating the data in a computer-readable form. Many of my colleagues at Berkeley provided useful discussion and criticisms. This work has been partly supported by a University of California Graduate Fellowship and the NSF grant AST-8118557.

\section{REFERENCES}

Abell, G. O. 1977, Ap. J., 213, 327.

Adams, M. T., Strom, K. M., and Strom, S. E. 1980, Ap. J., 238, 445.

Binggeli, B. 1982, Astr. Ap., 107, 338.

Brown, F. G. 1964, M.N.R.A.S., 127, 517 $1968, M N R$ A S 138, 527

Carter, D., and Metcalfe, N. 1980, M.N.R.A.S., 191, 325

Doroshkevich, A. G. 1973, Ap. Letters, 14, 11.

Doroshkevich, A. G., and Shandarin, S. F. 1974, Soviet Astr., 18, 24.

Godwin, J. G., Metcalfe, N., and Peach, J. V. 1983, M.N.R.A.S., 202, 113.

Gregory, S. A., and Thompson, L. A. 1978, Ap. J., 222, 784

Hawley, D. L., and Peebles, P. J. E. 1975, A.J., 80, 477.

Kaprandis, S., and Sullivan, W. T. 1983, Astr. Ap., 118, 33.

Kent, S. M., and Gunn, J. E. 1982, A.J., 87, 945 .

Koo, D. C. 1981, Ph.D. thesis, University of California, Berkeley.
MacGillivray, H. T., Dodd, R. J., McNally, B. V., and Corvin, H. G. 1982, M.N.R.A.S., 198, 605 .

Ozernoi, L. M. 1972, Soviet Astr., 15, 923. 1978 , in IAU Symposium 79, Large Scale Structure of the Universe, ed. M. S. Longair and J. Einasto (Dordrecht: Reidel), p. 427.

Palmer, P. L. 1983, M.N.R.A.S., 202, 561

Rood, H. J., and Baum, W. 1967, A.J., 72, 398.

Rood, H. J., Page, T. L., Kintner, E. C., and King, I. R. 1972, Ap. $J ., 175,627$.

Schipper, L., and King, I. R. 1978, Ap. J., 220, 798.

Shandarin, S. F. 1974, Soviet Astr., 18, 392.

Strom, S. E., and Strom, K. M. 1978, A.J., 83, 732.

Thompson, L. A. 1976, Ap. J., 209, 22.

Zeldovich, Ya. B. 1978, in IAU Symposium 79, Large Scale Structure of the Universe, ed. M. S. Longair and J. Einasto (Dordrecht: Reidel), p. 409.

S. DJoRgovski: Astronomy Department, University of California, Berkeley, CA 94720 